\title{
MAPEAMENTO DA OFERTA DE CURSOS \\ E VAGAS DOS PROGRAMAS DE RESIDÊNCIA \\ EM ENFERMAGEM NA BAHIA
}

\author{
MAPPING OF OFFERS OF COURSES AND \\ VACANCIES IN NURSING RESIDENCE \\ PROGRAMS IN BAHIA
}

\section{MAPEO DE LA OFERTA DE CURSOS Y VACANTES DE LOS PROGRAMAS DE RESIDENCIA EN ENFERMERÍA EN LA BAHIA}

\author{
Rosana Maria de Oliveira Silva ${ }^{1}$ \\ Rebeca Santos do Amaral de Souza ${ }^{2}$ \\ Lázaro Souza da Silva ${ }^{3}$ \\ Ana Lúcia Arcanjo Oliveira Cordeiro ${ }^{4}$ \\ Olga Maria Brito dos Santos \\ Josicelia Dumêt Fernandes ${ }^{6}$
}

Como citar este artigo: Silva RMO, Souza RSA, Silva LS, Cordeiro ALAO, Santos OMB, Fernandes JD. Mapeamento da oferta de cursos e vagas dos programas de residência em enfermagem na Bahia. Rev baiana enferm. 2018;32:e27505.

Objetivo: mapear a oferta de cursos e vagas dos programas de residência multiprofissional e em área profissional da saúde em enfermagem na Bahia. Método: estudo documental, quantitativo, cujas fontes de dados foram 15 editais de processo seletivo para programas de residência em enfermagem que descreveram as variáveis ano, número de programa, áreas de concentração, vagas, instituições de ensino superior vinculadas e modalidades dos programas. A coleta deu-se de 15 a 19 de janeiro de 2018. Resultados: foram identificadas 566 vagas distribuídas entre 19 áreas de concentração e ofertadas por oito instituições de ensino. No ano de 2014 houve a maior oferta de vagas 107 (18,9\%), em 2015 houve queda considerável de 57,9\% no número dessas vagas. Conclusão: os programas de residência multiprofissional expandiram-se em decorrência de reformulações das políticas de saúde e dos incentivos à formação de recursos humanos especializados, no entanto vem ocorrendo redução na oferta de vagas.

Descritores: Especialização. Internato não Médico. Educação. Ensino. Enfermagem.

Objective: map the offer of courses and vacancies in multiprofessional residency programs and in the professional health area in nursing in Babia. Method: documentary, quantitative study, whose data sources were 15 selective process edicts for nursing residency programs that described the variables year, number of program, concentration areas, vacancies, linked higher education institutions, and program modality. The collection took place from January 15 to 19, 2018. Results: 566 vacancies were identified distributed among 19 concentration areas and areas offered by

\footnotetext{
Enfermeira. Doutora em Enfermagem. Professora Adjunta IV da Escola de Enfermagem da Universidade Federal da Bahia. Salvador, Bahia, Brasil. Enfermeira. Salvador, Bahia, Brasil.

Enfermeiro. Mestre em Enfermagem. Salvador, Bahia, Brasil.

Enfermeira. Doutora em Enfermagem. Professora Adjunta da Escola de Enfermagem da Universidade Federal da Bahia. Salvador, Bahia, Brasil.

Enfermeira. Salvador, Bahia, Brasil. olgambs@hotmail.com

Enfermeira. Doutora em Enfermagem. Professora Emérita da Escola de Enfermagem da Universidade Federal da Bahia. Salvador, Bahia, Brasil.
} 
eight educational institutions. In 2014, there was the greatest number of vacancies, 107 (18.9\%); in 2015 there was a considerable fall of $57.9 \%$ in the number of these vacancies. Conclusion: multiprofessional residency programs have expanded due to reformulations of health policies and incentives to the formation of specialized human resources; however, there has been a reduction in the availability of vacancies.

Descriptors: Specialization. Nonmedical Internship. Education. Teaching. Nursing.

Objetivo: mapear la oferta de cursos y vacantes de programas de residencia multiprofesional y en área profesional de la salud en enfermería en la Babia, Brasil. Método: estudio documental, cuantitativo, cuyas fuentes de datos fueron 15 ediciones de proceso selectivo para programas de residencia en enfermería que describieron las variables año, número de programa, áreas de concentración, vacantes, instituciones de enseñanza superior vinculada $y$ modalidad de los programas. Recolección de 15 a 19 de enero de 2018. Resultados: se identificaron 566 vacantes distribuidas entre 19 áreas de concentración y ofrecidas por ocho instituciones de enseñanza. En 2014, hubo mayor oferta de vacantes 107 (18,9\%); en 2015, descenso considerable de 57,9\% en el número de vacantes. Conclusión: los programas de residencia multiprofesional se expandieron como consecuencia de reformulaciones de politicas de salud e incentivos a la formación de recursos humanos especializados, sin embargo, bubo reducción en la oferta de vacantes.

Descriptores: Especialización. Internado no Médico. Educación. Enseñanza. Enfermería.

\section{Introdução}

Os programas de residência para enfermeiros são oferecidos no Brasil desde a década de 1970, no entanto foram regulamentados enquanto modalidade de ensino de pós-graduação lato sensu em 2005, com a Lei n. 11.129, que cria a residência multiprofissional em saúde (RMS). É um programa de cooperação intersetorial para facilitar a inserção qualificada de jovens profissionais da saúde no mercado de trabalho e para atender às necessidades de recursos humanos do Sistema Único de Saúde (SUS) ${ }^{(1-2)}$.

Essas necessidades projetam-se em virtude das novas concepções a respeito do perfil profissional em saúde e sua atuação, a qual deve ser pautada nos princípios do SUS, de modo a contribuir para o fortalecimento desse sistema. Assim, a residência é uma referência para a formação dos trabalhadores da saúde, pois sua proposta é comprometida com o SUS e é transformadora, ao utilizar a educação permanente e a formação em serviço.

A modalidade de formação do programa de residência multiprofissional possibilita a construção e o intercâmbio do conhecimento articulado entre os diversos profissionais da área de saúde em um mesmo contexto de prática profissional. Isso a diferencia da modalidade de residências em áreas específicas da saúde ${ }^{(3-5)}$, nas quais a discussão dá-se na mesma vertente profissional. No entanto, existem similaridades entre as modalidades de residências e estas encontram-se, sobretudo, nos benefícios por elas proporcionados. Nesse sentido, preparam o profissional para o trabalho em centros de alta tecnologia, para ocupar cargos de gerência e liderança e, sobretudo, favorecem a inserção e retenção do trabalhador no mercado de trabalho ${ }^{(5-10)}$. Além disso, propiciam capacitação para o desenvolvimento de pesquisas e consequente melhoria na qualidade da assistência prestada nos serviços de saúde.

Diante das contribuições apresentadas por essa modalidade de ensino e com vistas ao aprimoramento dos programas e estímulo à sua expansão ao longo dos anos, legislações foram regulamentadas em relação à: duração e carga horária dos programas; organização, funcionamento e atribuições da Comissão de Residência Multiprofissional; instituição da Comissão Nacional de Residência Multiprofissional em Saúde, com as normas de funcionamento, atribuições e o sistema de Informação dessa comissão; e o Programa Nacional de Bolsas para as Residências ${ }^{(11-12)}$. Desse modo, ao longo dos anos, houve tendência expansionista dos programas, 
com aumento do número de vagas e de áreas de concentração.

Assim, ao revelar o avanço, o crescimento e/ou a redução de programas e do número de vagas, este estudo poderá contribuir para a Enfermagem, para as instituições parceiras e para o SUS.

Desse modo, questiona-se: Qual o mapeamento da oferta de cursos e vagas dos programas de residência multiprofissional e em área profissional da saúde em enfermagem na Bahia? O objetivo deste estudo é mapear a oferta de cursos e vagas dos programas de residência multiprofissional e em área profissional da saúde em enfermagem na Bahia.

\section{Método}

Trata-se de um estudo descritivo de base documental com abordagem quantitativa, que buscou os editais do processo seletivo para ingresso em programas de residência multiprofissional e em área profissional da saúde em enfermagem na Bahia, de 2006 a 2017. O recorte temporal refere-se ao ano de criação e regulamentação, em 2005, até o edital de 2017. A pesquisa constituiu-se no levantamento dos editais e caracterização dos programas de residência multiprofissional e em área profissional da saúde em enfermagem na perspectiva do movimento de expansão dessa modalidade de ensino no estado da Bahia.

As buscas foram realizadas no período de 15 a 19 de janeiro de 2018 na página on-line da Comissão Estadual da Residência Multiprofissional e em Área Profissional da Saúde (CEREMAPS), vinculada à Secretaria de Saúde do Estado da Bahia (Sesab), utilizando o termo "edital da residência multiprofissional e em área profissional" com o ano referente. Este portal direciona a busca para as páginas on-line das consultorias técnicas responsáveis pela execução dos editais das residências no estado da Bahia. Na etapa de coleta dos dados, todos os editais foram levantados nesse portal.

Foram delimitados como critérios de inclusão: editais de abertura de processo seletivo para ingresso em programas de residência multiprofissional e em área profissional da saúde com vagas para enfermeiros, no estado da Bahia, entre os anos de 2006 e 2017, e respectivas erratas encontradas. Como critério de exclusão: Editais em duplicata no momento da busca. Desse modo, selecionaram-se 15 editais. Ressalta-se que, nos anos de 2014, 2015 e 2016, foram publicados dois editais.

Após a seleção desses editais, foram coletados os dados especificamente referentes aos programas de residência que disponibilizaram vagas para enfermeiros. Nesse sentido, os dados contemplavam as seguintes variáveis: ano do edital, quantidade de programas para enfermeiros, quantidade e tipos de áreas de concentração para enfermeiros, quantidade de vagas para enfermeiros, unidades de ensino superior vinculadas e modalidade dos programas (multiprofissional ou em área profissional).

Os dados coletados foram sistematizados em planilhas no Microsoft Office Excel $2013^{\circledR}$, que permitiu posterior utilização de estatística descritiva simples com base na análise gráfica. Para melhor expressar o mapeamento da oferta de cursos e vagas dos programas de residência multiprofissional e em área profissional da saúde em enfermagem, os dados estão dispostos em gráficos e tabelas.

Este estudo foi realizado com dados de domínio público, não envolvendo seres humanos, contudo, por ser o recorte de um projeto de pesquisa financiado, intitulado "Trajetória Profissional de Enfermeiros Egressos dos Programas de Residência Multiprofissional e em Área Profissional da Saúde", o estudo dispõe de aprovação de comitê de ética, número de protocolo CAAE 55876516.8.0000.5028.

\section{Resultados}

Os resultados permitiram identificar-se que, entre 2006 e 2014, o número de programas de residência e áreas de concentração apresentaram tendência de aumento, com destaque para o ano de 2014 cujo edital ofertou 18 áreas de concentração em 21 programas. No período de 11 anos, 
foram oferecidas 566 vagas para enfermeiros na Bahia. O Gráfico 1 apresenta a distribuição do total de programas, áreas de concentração e vagas ofertadas.

Gráfico 1 - Distribuição dos programas, áreas de concentração e número de vagas de residências para enfermeiros. Salvador, Bahia, Brasil - 2006-2017

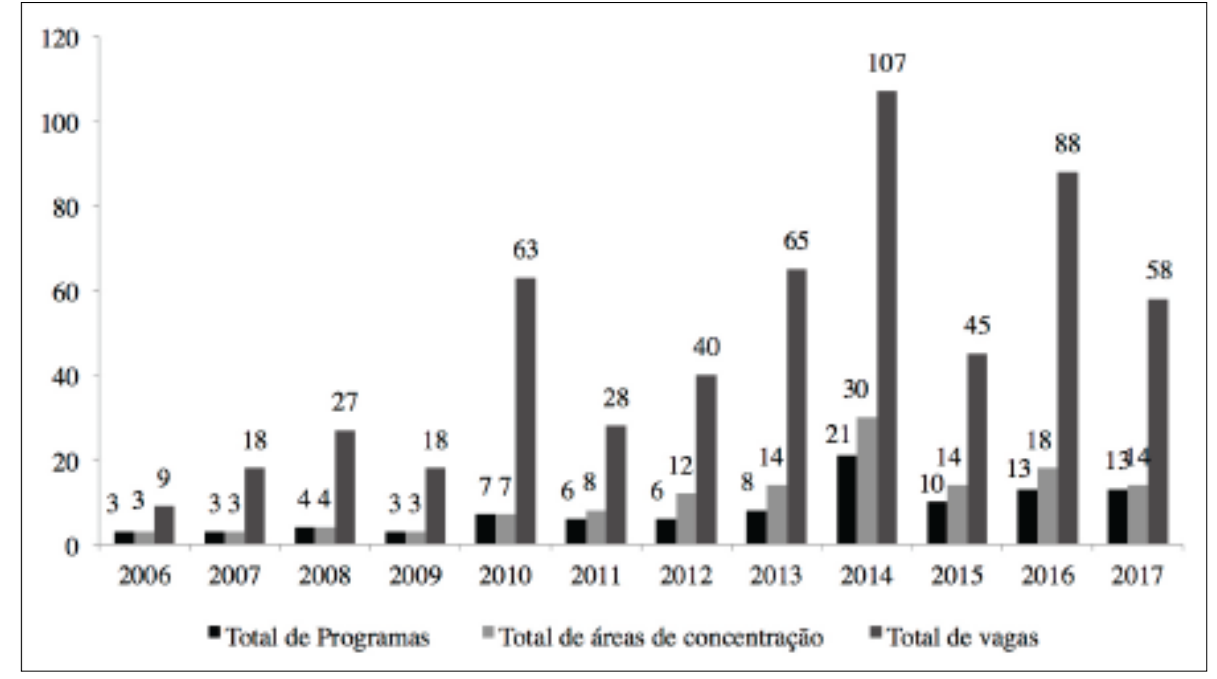

Fonte: Elaboração própria.

Na Tabela 1 foram elencadas as 19 áreas de concentração dos programas, quantas vezes foram oferecidas e o número de vagas disponibilizadas para cada área ao longo desses 11 anos.

Tabela 1 - Área de concentração, ocorrência e número de vagas das áreas de concentração dos programas de residência para enfermeiros. Salvador, Bahia, Brasil - 2006-2017

\begin{tabular}{l|c|c}
\hline Área de Concentração & $\begin{array}{c}\text { Ocorrência } \\
\mathbf{n}(\mathbf{\%})\end{array}$ & $\begin{array}{c}\text { Vagas } \\
\mathbf{n}(\mathbf{\%})\end{array}$ \\
\hline Saúde da Família & $31(23,5)$ & $111(16,6)$ \\
Terapia intensiva & $26(19,7)$ & $256(45,2)$ \\
Saúde Mental & $13(9,8)$ & $19(3,4)$ \\
Saúde da Criança e do Adolescente & $13(9,8)$ & $18(3,2)$ \\
Saúde do Adulto & $8(6,1)$ & $8(1,4)$ \\
Oncologia & $6(4,5)$ & $13(2,3)$ \\
Cardiologia & $6(4,5)$ & $19(3,4)$ \\
Saúde do Idoso & $4(3)$ & $8(1,4)$ \\
Urgências e Emergências & $4(3)$ & $16(2,8)$ \\
Obstetrícia & $4(3)$ & $54(9,5)$ \\
Clínica da Pessoa e da Família & $4(3)$ & $8(1,4)$ \\
Planejamento e Gestão em Saúde & $3(2,3)$ & $7(1,3)$ \\
Nefrologia & $3(2,3)$ & $8(1,4)$ \\
Saúde Materno-Infantil & $2(1,5)$ & $7(1,3)$ \\
Atenção à Saúde Hospitalar & $1(0,7)$ & $6(1,2)$ \\
Saúde Coletiva & $1(0,7)$ & $2(0,3)$ \\
Neurologia & $1(0,7)$ & $2(0,3)$ \\
Neonatologia & $1(0,7)$ & $2(0,3)$ \\
Vigilância em saúde & $1(0,7)$ & $2(0,3)$ \\
\hline
\end{tabular}

Fonte: Elaboração própria. 
Na Tabela 2, estão identificados as Instituições de Ensino Superior e o número de vagas dos programas de residência destinados a enfermeiros por ano em que foram ofertadas.

Tabela 2 - Distribuição do número de vagas dos programas de residência para enfermeiros segundo Instituições de Ensino Superior. Salvador, Bahia, Brasil - 2006-2017

\begin{tabular}{|c|c|c|c|c|c|c|c|c|}
\hline Ano Unidade & UFBA & UNEB & EESP & BAHIANA & UNIFACS & FAMAM & UNIVASF & FPS \\
\hline 2006 & 6 & - & - & 1 & - & - & 2 & - \\
\hline 2007 & 14 & - & - & 2 & - & - & 2 & - \\
\hline 2008 & 23 & - & - & 2 & - & - & 2 & - \\
\hline 2009 & 14 & - & - & 2 & - & - & 2 & - \\
\hline 2010 & 47 & - & 10 & 4 & - & - & 2 & - \\
\hline 2011 & 22 & - & - & 4 & - & - & 2 & - \\
\hline 2012 & 21 & 13 & - & 4 & - & - & 2 & - \\
\hline 2013 & 29 & 14 & - & 4 & - & 14 & 2 & 2 \\
\hline 2014 & 47 & 18 & - & 6 & 14 & 14 & 4 & 4 \\
\hline 2015 & 13 & 9 & 15 & 4 & 2 & - & 2 & - \\
\hline 2016 & 42 & 14 & 14 & 2 & 14 & - & 2 & - \\
\hline 2017 & 26 & 14 & 10 & 4 & 2 & - & 2 & - \\
\hline Total & 304 & 82 & 49 & 39 & 32 & 28 & 26 & 6 \\
\hline
\end{tabular}

Fonte: Elaboração própria.

Nota: Sinal convencional utilizado:

- Dado numérico igual a zero não resultante de arredondamento.

Legenda: UFBA - Universidade Federal da Bahia; UNEB - Universidade do Estado da Bahia; EESP - Escola Estadual de Saúde Pública Professor Francisco Peixoto Magalhães Neto; BAHIANA - Escola Bahiana de Medicina e Saúde Pública; UNIFACS - Universidade Salvador; FAMAM - Faculdade Maria Milza; UNIVASF - Universidade Federal do Vale do São Francisco; EPS - Faculdade Pernambucana de Saúde.

O Gráfico 2 apresenta a distribuição dos programas de residência de acordo com a modalidade oferecida para o enfermeiro, na Bahia, no período estudado.

Gráfico 2 - Distribuição dos programas de residência de acordo com a modalidade oferecida para enfermeiros - 2006-2017

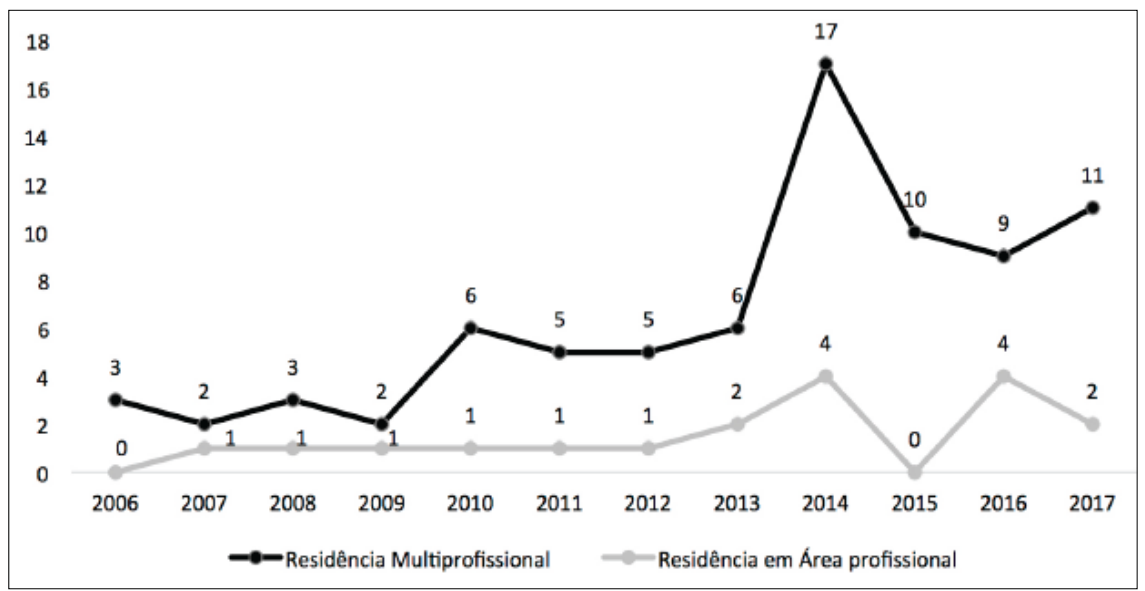

Fonte: Elaboração própria. 


\section{Discussão}

Os dados apresentados evidenciaram que a oferta de programas e áreas de concentração de residência em enfermagem na Bahia oscilou no período de 2006 a 2017, contudo apresentou uma tendência de crescimento que atingiu o ápice em 2014. No ano de 2014, foram disponibilizadas 107 (18,9\%) vagas de residência em enfermagem, o maior quantitativo observado no período analisado. No entanto, em 2015, ocorreu um declínio expressivo nessa disponibilização. Ainda no âmbito da oferta de vagas, a Universidade Federal da Bahia destacou-se, ofertando 304 (53,7\%) vagas nesses anos. Quanto à área de concentração, a de saúde da família foi a mais oferecida. Por fim, os programas com modalidade multiprofissional foram mais disponibilizados 79 (81,5\%) em relação aos programas em áreas profissionais 18 (18,5\%).

A expansão apresentada pelos programas de residência em enfermagem na Bahia entre 2006 e 2017 está diretamente relacionada ao pronunciado processo de expansão dos cursos de graduação em enfermagem, que teve início em 1991. Esse processo foi marcado pelo aumento de escolas de enfermagem e consequente aumento do número de egressos dos cursos de enfermagem $^{(13-14)}$.

Fatores que propiciaram essas mudanças no cenário educativo da enfermagem em âmbitos de graduação e pós-graduação foram as reformulações no setor saúde, marcadas pela necessidade nacional de desenvolvimento de recursos humanos aptos e especializados a atuarem no SUS, com vistas ao fortalecimento desse sistema. Isso evidenciou mudanças na natureza e no processo de trabalho dos profissionais, ao ter como consequência, no âmbito da residência, maior busca por essa modalidade de especialização e aumento dos incentivos governamentais para os cursos e programas.

Dessa forma, a consolidação dos programas de residência multiprofissional e em área profissional da saúde constitui-se em um movimento político importante para propor mudanças na forma de produzir saúde com foco na interdisciplinaridade, por meio da presença das universidades nas unidades de saúde, para uma prática transformadora que atenda às necessidades locais de seus usuários ${ }^{(15)}$.

Quanto à área de concentração, observou-se que a mais ofertada para enfermeiros foi a de saúde da família, priorizando a integralidade e fortalecendo a atenção básica. Isso evidencia o potencial desses programas como proposta fortalecedora do sistema de saúde, refletindo as novas reformulações na formação de profissionais de diversas áreas, pautadas na integralidade e articulação das ações para o atendimento à saúde da população ${ }^{(16)}$.

As mudanças na legislação e na organização dos serviços de saúde também concorreram para o aumento da área de concentração e consequentemente do número de vagas em saúde da família, como aponta a nova Portaria n. 2.488/2011, a qual aprova a Política Nacional da Atenção Básica (PNAB). Essa aprovação tornou necessário o aumento do número de profissionais da área, com formação acadêmica específica para atender ao exposto pela política ${ }^{(17)}$. Esse fato pôde ser observado com a publicação de editais, nos últimos três anos, pela Escola de Saúde Pública do Estado da Bahia (EESP), com vagas unicamente para a área de saúde da família.

Em relação ao número de vagas, observa-se maior crescimento na área de Terapia Intensiva, que representa $256(45,2 \%)$ do total de vagas das 19 áreas de concentração existentes. Esse aumento no número de vagas para terapia intensiva decorre do fato de esta especialidade necessitar de profissionais altamente qualificados, para estarem aptos a trabalhar prestando assistência com qualidade, já que o trabalho do enfermeiro em uma Unidade de Terapia Intensiva (UTI) é caracterizado por atividades que exigem aperfeiçoamento constante de competências necessárias para o cuidado e o manejo do doente crítico $^{(18)}$. Além disso, relaciona-se a suprir uma demanda maior de profissionais, tanto pela alta rotatividade, quanto por exigências legais em relação ao dimensionamento de pessoal, no qual o número de enfermeiros em uma UTI corresponde a $56 \%$ de todo o recurso 
humano necessário para funcionamento de uma unidade $^{(19)}$.

Outro aspecto que motivou a expansão dessa área foi o fato de ter sido uma das prioritárias de formação de recursos humanos para o SUS, tanto pela expansão do número de leitos públicos e privados em UTI quanto pelas mudanças advindas com a RDC n. 7/2010, no que tange aos padrões mínimos de profissionais para funcionamento das UTIs no Brasil. Com isso, aumentou o investimento e a ampliação de vagas e programas ${ }^{(20)}$.

Diante do potencial dos programas de residência multiprofissional e em área profissional da saúde, o governo brasileiro fomentou investimentos visando o fortalecimento e a expansão desse modelo de ensino no país. No período de 2007 a 2009, os Ministérios da Saúde e da Educação investiram aproximadamente 180 milhões de reais nesses programas. Em 2010 foi implementado o Programa de Residência Integrada Multiprofissional em Saúde da rede de Hospitais Universitários Federais, para o qual foram disponibilizadas 500 bolsas, a um custo anual estimado de 11 milhões de reais. No ano seguinte, a oferta de bolsas para a residência multiprofissional duplicou ${ }^{(21-22)}$.

Entretanto, observou-se, neste estudo, que a quantidade de bolsas ofertadas a partir de 2015 decresceu, com queda de 62 unidades $(57,9 \%)$ entre 2014 e 2015. Isso reflete o momento político do Brasil, caracterizado por forte crise econômica, com cortes orçamentários em todos os setores do governo, inclusive na educação. Em 2016, a oferta de vagas cresceu 43 unidades após a queda em 2015, o que revela as tentativas de saída da crise e retorno dos investimentos em educação, contudo, no ano de 2017, não se manteve esse crescimento devido ao cenário político atual de desmonte do SUS, com a privatização dos serviços de saúde pelo corporativismo de manutenção de um modelo hegemônicos biomédico que se opõe a toda e qualquer mudança nos perfis dos profissionais de saúde para a não edificação do SUS ${ }^{(15)}$.
Além disso, a Proposta de Emenda Constitucional (PEC) n. 95/2016, que instituiu o novo regime de gastos públicos, também conhecida como "PEC dos Gastos", ao ter como propósito o congelamento dos investimentos em educação e saúde, promoverá a manutenção dessa redução na formação de recursos humanos para o SUS ao longo dos anos de sua vigência ${ }^{(23)}$.

Com relação ao número de vagas/bolsas por Instituições de Ensino, observa-se um quantitativo expressivo disponibilizado pela Universidade Federal da Bahia (UFBA), se comparado às demais instituições. Isso pode estar ligado ao fato de que a UFBA foi a primeira instituição pública de ensino superior na Bahia e pioneira na pós-graduação lato e stricto sensu, e também na oferta do primeiro curso de especialização na modalidade residência na Bahia e no Brasil. Também influenciou para o seu destaque na oferta de vagas desses programas a sua localização, com o maior campi na capital baiana, onde há maior articulação com os serviços disponibilizados para o campo de prática.

Com este estudo, percebeu-se que, ao longo dos anos, os programas multiprofissionais destacaram-se quando comparados aos programas em áreas profissionais da saúde. Isso se deve ao fato de a formação com profissionais de todas as áreas da saúde possibilitar a capacitação voltada à integralidade da assistência, com impacto na consolidação de um sistema de saúde humanizado ${ }^{(24)}$.

Considera-se como limitações deste estudo a impossibilidade de generalizar esses achados para as diversas regiões do Brasil, como também o aprofundamento da análise em relação aos motivos intrínsecos e extrínsecos que levaram ao aumento e à diminuição do número de vagas e programas de residência, ao atender somente o caráter descritivo proposto. Serve, entretanto, como sugestão para novos estudos, na ampliação de discussão que atinja um caráter analítico e possa dar respostas mais aprofundadas aos motivos de expansão e retração no número de vagas, bolsas e programas de residência. 


\section{Conclusão}

A oferta de cursos e vagas dos programas de residência multiprofissional e em área profissional da saúde em Enfermagem no estado da Bahia ocorreu com tendência expansionista ao longo dos anos de 2006 a 2014 e declínio a partir de 2015. Nesse período de 11 anos analisados (2006 a 2017), foram oferecidas 566 vagas. Observou-se que, no ano de 2014, foi ofertado o maior número de vagas (107), em 18 áreas de concentração de 21 programas. As áreas de concentração com maior quantitativo de vagas foram: Saúde da Família, com 31 ocorrências e 111 vagas; e a de Terapia Intensiva, com 26 ocorrências e 256 vagas. Dentre as instituições de ensino, a UFBA foi a que mais se destacou no oferecimento de número de vagas: 304. A modalidade com maior número de programas foi a Residência Multiprofissional.

Este estudo apresenta implicação na área da enfermagem e dos órgãos responsáveis pela formação e desenvolvimento de recursos humanos para a saúde, dando visibilidade ao real crescimento e/ou redução dos programas, de forma a subsidiar a criação de outras áreas, ampliação do número de vagas e de parcerias para atender às necessidades do SUS.

Pode-se inferir, contudo, que a política de cortes orçamentários praticada pelo governo brasileiro na atualidade pode acarretar a descontinuidade do investimento na formação de recursos humanos para o SUS e tornar-se um dos elementos para o seu desmonte.

\section{Colaborações:}

1. concepção, projeto, análise e interpretação dos dados: Rosana Maria de Oliveira Silva, Rebeca Santos do Amaral de Souza e Lázaro Souza da Silva;

2. redação do artigo e revisão crítica relevante do conteúdo intelectual: Rosana Maria de Oliveira Silva, Rebeca Santos do Amaral de Souza, Lázaro Souza da Silva, Ana Lúcia Arcanjo Oliveira Cordeiro, Olga Maria Brito dos Santos e Josicelia Dumêt Fernandes;
3. aprovação final da versão a ser publicada: Rosana Maria de Oliveira Silva, Rebeca Santos do Amaral de Souza, Lázaro Souza da Silva, Ana Lúcia Arcanjo Oliveira Cordeiro, Olga Maria Brito dos Santos e Josicelia Dumêt Fernandes.

\section{Referências}

1. Martins GDM, Caregnato RCA, Barroso VLM, Ribas DCP. Implementation of multi-professional healthcare residency at a Federal University: historical trajectory. Rev Gaúcha Enferm [Internet]. 2016 [cited 2018 Mar 9];37(3):1-8. Available from: DOI: $10.1590 / 1983-1447.2016 .03 .57046$

2. Brasil. Lei n. 11.129, de 30 de junho de 2005. Institui o Programa Nacional de Inclusão de Jovens - ProJovem, cria o Conselho Nacional da Juventude - CNJ e a Secretaria Nacional de Juventude, altera as Leis n. 10.683 e 10.429 e dá outras providências [Internet]. Brasília; 2005 [cited 2018 Mar 9]. Available from: http://www.camara. gov.br/sileg/integras/535652.pdf

3. Casanova IA, Batista NA, Ruiz-Moreno L. Training for teamwork in multidisciplinary residency in health. Abc Health Sci [Internet]. 2015 [cited 2018 Mar 9];40(3):229-33. Available from: DOI: 10.7322/ abcshs.v40i3.800

4. Fernandes MNS, Beck CLC, Weiller TH, Viero V, Freitas PH, Prestes FC. Suffering and pleasure in the process of forming multidisciplinary health residents. Rev Gaúcha Enferm [Internet]. 2015 [cited 2018 Mar 10];36(4):90-7. Available from: DOI: $10.1590 / 1983-1447.2015 .04 .50300$

5. Silva CT, Terra MG, Kruse MHL, Companogara S, Xavier MS. Multi-professional residency as an intercessor for continuing education in health. Texto Contexto Enferm [Internet]. 2016 [cited 2018 Mar 10];25(1):1-9. Available from: DOI: 10.1590/0104-0707201600002760014

6. Magnabosco G, Haddad MCL, Vannuchi MTO, Rossaneis MA, Silva LGC. Opinion of former residency students in nursing management. Semina cienc biol saude [Internet]. 2015 [cited 2018 Mar 17];36(1):73-80. Available from: DOI: 10.5433/1679-0367.2015v36n1Suplp73

7. Garrison FW, Dearmon V, Graves RJ. Working smarter: Building a better nurse residency program. Nurs Manag [Internet]. 2017 [cited 2018 Mar 17];48(3):50-4. Available from: DOI: 10.1097/01. numa.0000512898.76048.0b 
8. Goode CJ, Lynn MR, McElroy D, Bednash GD, Murray B. Lessons Learned From 10 Years of Research on a Post-Baccalaureate Nurse Residency Program. J Nurs Adm [Internet]. 2013 [cited 2018 Mar 17];43(2):73-9. Available from: DOI: 10.1097/ nna.0b013e31827f205c

9. Johnson A, Salisbury H, Johannsson M, Barajas K. Emergency Nurse Residency Program Evaluation. J Nurses Prof Dev [Internet]. 2013 [cited 2018 Mar 17];29(5):233-7. Available from: DOI: 10.1097/01. nnd.0000433908.82754.10

10. Kram SL, Wilson J. Nurse residency program attracts and retains novice nurses. Nursing [Internet]. 2016 [cited 2018 Mar 17];46(2):15-6. Available from: DOI: 10.1097/01.nurse.0000476245.39588.ff

11. Brasil. Portaria Interministerial n. 1.320 , de 11 de novembro de 2010. Dispõe sobre a estrutura, organização e funcionamento da Comissão Nacional de Residência Multiprofissional em Saúde [Internet]. Brasília; 2010 [cited 2018 Mar 25]. Available from: http://www.ufjf.br/huresidencias/ files/2017/07/Portaria-n\%C2\%B01320.pdf

12. Brasil. Resolução CNRMS n. 5 , de 7 de novembro de 2014. Dispõe sobre a duração e a carga horária dos Programas de Residência em Área Profissional da Saúde nas modalidades multiprofissional e uniprofissional e sobre a avaliação e frequência dos profissionais de saúde residentes [Internet]. Brasília; 2014 [cited 2018 Mar 25]. Available from: https:// www.legisweb.com.br/legislacao/?id=276672

13. Fernandes JD, Teixeira GAS, Silva MG, Florêncio RMS, Silva RMO, Rosa DOS. Expansion of higher education in Brazil: increase in the number of Undergraduate Nursing courses. Rev Latino-am Enfermagem [Internet]. 2013 [cited 2018 Apr 3];3(21):1-8. Available from: DOI: 10.1590/ S0104-11692013000300004

14. Erdmann AL, Fernandes JD, Teixeira GAS. Panorama da educação em enfermagem no Brasil: graduação e pós-graduação. Enferm Foco [Internet]. 2015 [cited 2018 Apr 3];89-93. Available from: http://revista.portalcofen.gov.br/index.php/ enfermagem/article/view/91

15. Meneses JR, Ceccim RB, Martins GC, Meira IFF, Silva VM. Residência em saúde: os movimentos que as sustentam. In: Ceccim RB, Meneses LBA, Soares VL, Pereira AJ, Meneses JR, Rocha RCS, et al. Formação de formadores para residências em saúde: corpo docente-assistencial em experiência viva. Porto Alegre, RS: Rede UNIDA; 2018. Série Vivências em Educação na Saúde. p. 33-48.
16. Domingos CM, Nunes EFPA, Carvalho BG. Potential of multiprofessional residency for family healthcare: the view of healthcare workers. Interface Comunic Saúde Educ [Internet]. 2015 [cited 2018 Apr 5];19(55):1221-32. Available from: DOI: $10.1590 / 1807-57622014.0653$

17. Brasil. Ministério da Saúde. Portaria n. 2.488, de 21 de outubro de 2011. Aprova a Política Nacional de Atenção Básica, estabelecendo a revisão de diretrizes e normas para a organização da Atenção Básica, para a Estratégia Saúde da Família (ESF) e o Programa de Agentes Comunitários de Saúde (PACS) [Internet]. Brasília; 2011 [cited 2018 Nov 24]. Available from: http://bvsms.saude.gov.br/bvs/ saudelegis/gm/2011/prt2488_21_10_2011.html

18. Frota LA, Camponogara S, Arbiot EL, Tolfo F, Beck CLC, Freitas EO. The nurse's visibility in intensive care units: perceptions of workers. Rev Eletr Enferm [Internet]. 2015 [cited 2018 May 7];17(3):1-8. Available from: DOI: 10.5216/ree.v17i3.31608

19. Camelo SHH. Professional competences of nurse to work in Intensive Care Units: an integrative review. Rev Latino-Am Enfermagem [Internet]. 2012 [cited 2018 May 7];20(1):192-200. Available from: DOI: 10.1590/S0104-11692012000100025

20. Brasil. Ministério da Saúde. Resolução RDC n. 7, de 24 de fevereiro de 2010. Dispõe sobre os requisitos mínimos para funcionamento de Unidades de Terapia Intensiva e dá outras providências [Internet]. Brasília; 2010 [cited 2018 Nov 24]. Available from: http://www.saude.mg.gov.br/images/documentos/ RDC-7_ANVISA\%20240210.pdf

21. Brasil. Relatório de Atividades da Comissão Nacional de Residência Multiprofissional em Saúde - CNRMS Exercício 2007/2009 [Internet]. Brasília; 2009 [cited 2018 May 10]. Available from: http://www.sbfa.org.br/portal/pdf/Relatorio\%20 Atividades\%20CNRMS\%202007\%202009.pdf

22. Mioto RCT, Alves FL, Caetano PS, Dal Prá KR. The multiprofessional residence programs in healthcare: the experience of the Federal University at Santa Catarina. Serv Soc Saúde [Internet]. 2012 [cited 2018 May 10];11(2):185-209. Available from: DOI: $10.20396 /$ sss.v11i2.8635288

23. Brasil. Presidência da República. Casa Civil. Emenda Constitucional n. 95, de 15 de dezembro de 2016. Altera o Ato das Disposições Constitucionais Transitórias, para instituir o Novo Regime Fiscal, e dá outras providências [Internet]. Brasília; 2016 [cited 2018 Nov 24]. Available from: http://www. 
planalto.gov.br/ccivil_03/constituicao/emendas/ emc/emc95.htm

24. Vendruscolo C, Prado ML, Kleba ME. Formation of human resources in the field of health in Brazil: an integrative review. Educ Rev [Internet]. 2014 [cited
2018 May 14];30(1):215-44. Available from: DOI: 10.1590/S0102-46982014000100009

Recebido: 31 de julho de 2018 Aprovado: 29 de novembro de 2018 Publicado: 28 de dezembro de 2018

A Revista Baiana de Enfermagem utiliza a Licença Creative Commons - Atribuição-NãoComercial 4.0 Internacional. https://creativecommons.org/licenses/by-nc/4.0/ Este artigo é de acesso aberto distribuído sob os termos da Licença Creative Commons (CC BY-NC). Esta licença permite que outros remixem, adaptem e criem a partir do seu trabalho para fins não comerciais. Embora os novos trabalhos tenham de lhe atribuir o devido crédito e não possam ser usados para fins comerciais, os usuários não têm de licenciar esses trabalhos derivados sob os mesmos termos. 